\title{
Nuclear and extended spectra of NGC 1068 - II. Near-infrared stellar population synthesis
}

\author{
Lucimara P. Martins, ${ }^{1 \star}$ Rogério Riffel, ${ }^{2}$ Alberto Rodríguez-Ardila, ${ }^{3}$ Ruth Gruenwald ${ }^{4}$ \\ and Ronaldo de Souza ${ }^{4}$ \\ ${ }^{1} N A T$ - Universidade Cruzeiro do Sul, Rua Galvao Bueno, 868 São Paulo, SP, Brazil \\ ${ }^{2}$ Universidade Federal do Rio Grande do Sul, IF, Departamento de Astronomia, CP 15051, 91501-970 Porto Alegre, RS, Brazil \\ ${ }^{3}$ Laboratório Nacional de Astrofísica/MCT, Rua dos Estados Unidos 154, CEP 37501-064 Itajubá, MG, Brazil \\ ${ }^{4}$ Instituto Astronômico e Geofísico, USP, Rua do Matão, 1226 São Paulo, SP, Brazil
}

Accepted 2010 April 7. Received 2010 April 7; in original form 2009 December 17

\begin{abstract}
We performed stellar population synthesis on the nuclear and extended regions of NGC 1068 by means of near-infrared spectroscopy to disentangle their spectral energy distribution components. This is the first time that such a technique is applied to the whole $0.8-2.4 \mu \mathrm{m}$ wavelength interval in this galaxy. NGC 1068 is one of the nearest and probably the most studied Seyfert 2 galaxy, becoming an excellent laboratory to study the interaction between black holes, the jets that they can produce and the medium in which they propagate. Our main result is that traces of young stellar population are found at $\sim 100 \mathrm{pc}$ south of the nucleus. The contribution of a power-law continuum in the centre is about 25 per cent, which is expected if the light is scattered from a Seyfert 1 nucleus. We find peaks in the contribution of the featureless continuum about $100-150 \mathrm{pc}$ from the nucleus on both sides. They might be associated with regions where the jet encounters dense clouds. Further support to this scenario is given by the peaks of hot dust distribution found around these same regions and the $\mathrm{H}_{2}$ emission-line profile, leading us to propose that the peaks might be associated to regions where stars are being formed. Hot dust also has an important contribution to the nuclear region, reinforcing the idea of the presence of a dense, circumnuclear torus in this galaxy. Cold dust appears mostly in the south direction, which supports the view that the south-west emission is behind the plane of the galaxy and is extinguished very likely by dust in the plane. Intermediate-age stellar population contributes significantly to the continuum, especially in the inner $200 \mathrm{pc}$.
\end{abstract}

Key words: galaxies: active - galaxies: individual: NGC 1068 - galaxies: Seyfert - infrared: galaxies.

\section{INTRODUCTION}

The coexistence of black holes and starburst clusters is known to exist in many galaxies, and there are many evidences that suggest a connection between these phenomena. Many studies point out that both the active nucleus and starbursts might be related to gas inflow, probably triggered by an axis-asymmetry perturbation like bars, mergers or tidal interactions (Shlosman, Frank \& Belgmann 1989; Shlosman, Belgmann \& Frank 1990; Maiolino et al. 1997; Knapen, Shlosman \& Peletier 2000; Fathi et al. 2006; Riffel et al. 2008). In addition, one of the most intriguing research areas in contemporary extragalactic astrophysics involves the study of the

\footnotetext{
^E-mail: lucimara.martins@ cruzeirodosul.edu.br
}

interplay between nuclear black holes, the jets produced by them and the interstellar (ISM)/intergalactic medium in which they propagate. These jets can have a considerable impact on this medium. One aspect of jet-ISM interaction is that it can trigger star formation. Such jet-induced star formation is considered a possible mechanism to explain the ultraviolet (UV) continuum emission observed in the host galaxies of distant radio sources and the 'alignment effect' between the radio emission and this continuum (Rees 1989). Although this effect might play a very important role in high- $z$ radio galaxies, detecting and studying the jet-ISM interaction in them is very challenging. Because of the observational problems, it is important to find nearby examples of this kind of interaction where a detailed study can be carried out.

NGC 1068 is an ideal object in this case. It is one of the nearest and probably the most intensely studied Seyfert 2 galaxy. 
Observations in all wavelength bands from radio to hard X-rays have formed a uniquely detailed picture of this source. NGC 1068 hosts a prominent narrow-line region that is approximately cospatial with a linear radio source with two lobes (Wilson \& Ulvestad 1983). Star formation activity coexistent with the active galactic nucleus (AGN) was detected on both larger (e.g. Telesco \& Decher 1988) and smaller scales (Macchetto et al. 1994; Thatte et al. 1997). However, the link between all the processes is still under debate.

The near-infrared (NIR) region is particularly interesting to help unveiling this link because it is accessible to ground-based telescopes and, at the same time, able to probe highly obscured sources. However, tracking the star formation in the NIR is not simple (Origlia \& Oliva 2000) although recent studies exploring this region have already shown its strong potential at detecting intermediate-age stellar population not easily tracked in the optical without ambiguity (e.g. Davies et al. 2007; Riffel et al. 2009). At NIR wavelengths stellar photospheres usually remain the dominant sources of light, and galaxy spectra are shaped by red supergiants shortly after starbursts, and then by giants of the first and asymptotic giant branches (AGB).

AGB stars are rare members of stellar populations. However, they are among the most luminous cool stars and can therefore be detected sometimes even individually in galaxies. The thermally pulsating AGB (TP-AGB) stars leave a unique fingerprint on the integrated spectra, like the 1.1- $\mu \mathrm{m} \mathrm{CN}$ band (Maraston 2005; Riffel et al. 2007, 2009). Hence when detected, they can help to determine the age of the stellar population through the integrated light. The contribution of this stellar phase in stellar population models has been recently included in both the energetics and the spectral features (Maraston 2005). In particular these models employ empirical spectra of oxygen-rich and carbon stars (Lançon \& Wood 2000), which are able to foresee characteristic NIR absorption features.

With this in mind, we present here for the first time in the literature a detailed fitting of the continuum emission components in the 0.8-2.4 $\mu \mathrm{m}$ interval of NGC 1068 across the central 15 arcsec $(\sim 1100 \mathrm{pc})$ of this source. The main purpose is to determine the fraction with which the different components contribute to the observed integrated light and how they are related to each other. The paper is structured as follows. In Section 2 we describe the observations. In Section 3 we describe the fitting method and in Section 4 the results are presented and discussed. Final remarks are given in Section 6.

\section{THE OBSERVATIONS}

The spectra were obtained at the NASA 3-m Infrared Telescope Facility (IRTF) in 2007 October 30. The SpeX spectrograph (Rayner et al. 2003) was used in the short cross-dispersed mode (SXD; $0.8-2.4 \mu \mathrm{m})$. The employed detector consisted of a $1024 \times 1024$ ALADDIN 3 InSb array with a spatial scale of $0.15 \operatorname{arcsec~pixel}^{-1}$. A $0.8 \times 15-\operatorname{arcsec}^{2}$ slit oriented in the north-south direction was used, providing a spectral resolution of $360 \mathrm{~km} \mathrm{~s}^{-1}$. For more details about the instrumental configuration see Martins et al. (2010, hereafter Paper I).

Fig. 1 shows the position of the slit superimposed on the galaxy contours obtained from Galliano et al. (2003). The grey contours show the 6-cm emission (Gallimore et al. 1996) and the red dotted contours show the 20- $\mu \mathrm{m}$ image (Alloin et al. 2000). For NGC 1068, 17 extractions were made along the spatial direction: one centred at the peak of light distribution (nuc) and eight more at each side of it (apertures 01-08 in the south direction and 09-16 in the north direction). Figs 5-7 of Paper I show the individual extractions

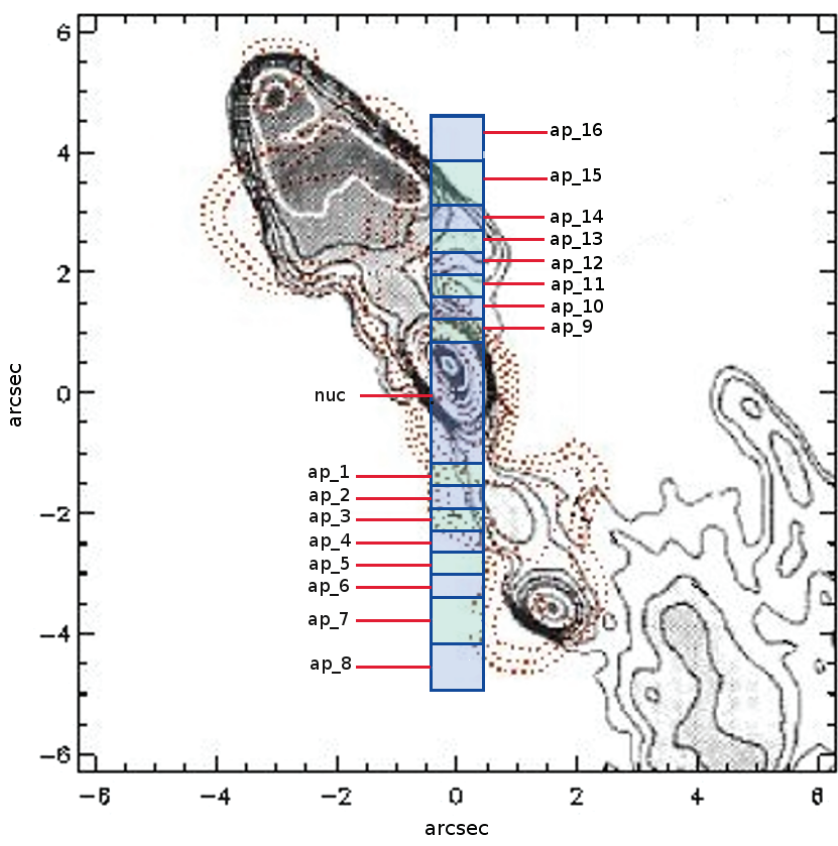

Figure 1. NGC 1068 contours obtained from Galliano et al. (2003) with the position of the IRTF slit superimposed. The grey contours show the 6-cm emission (Gallimore et al. 1996) and the red dotted contours show the 20- $\mu \mathrm{m}$ image (Alloin et al. 2000).

along the spatial direction as well as the most important emission and absorption features relevant to this paper.

\section{SPECTRAL SYNTHESIS}

Our main goal is to study the NIR spectral energy distribution components of NGC 1068 and their variations across the central $15 \mathrm{arcsec}$. For this purpose we fit the underlying continuum between 0.8 and $2.4 \mu \mathrm{m}$ applying the same method described in Riffel et al. (2009)

The spectral synthesis is done using the code STARLIGHT (Cid Fernandes et al. 2004, 2005a; Mateus et al. 2006; Asari et al. 2007; Cid Fernandes et al. 2009). STARLIGHT mixes computational techniques originally developed for semi-empirical population synthesis with ingredients from evolutionary synthesis models. Basically the code fits an observed spectrum $\mathrm{O}_{\lambda}$ with a combination, in different proportions, of a number of simple stellar populations (SSPs). Due to the fact that the Maraston (2005) models include the effect of the TP-AGB phase, crucial to model the stellar population in the NIR, we use these SSP models as the base set for STARLIGHT. The SSPs used in this paper cover 12 ages, $t=0.01,0.03,0.05,0.1,0.2,0.5$, $0.7,1,2,5,9$ and $13 \mathrm{Gyr}$, and four metallicities, $Z=0.02,0.5,1$ and $2 \mathrm{Z}_{\odot}$, summing up 48 SSPs.

In addition, in trying to describe the continuum of an AGN, the central engine cannot be ignored, even outside the nucleus. The stellar population alone cannot account for the bump in the $K$ band, clearly seen in most of the apertures. Besides that, studies have shown the presence of scattered light in NGC 1068 (e.g. Miller, Goodrich \& Mathews 1991; Inglis et al. 1995; Simpson et al. 2002). Usually this component is represented by a featureless continuum (FC; e.g. Koski 1978) of power-law form that follows the expression $F_{v} \propto v^{-1.5}$. (We have also tested other indexes for the power law - see Section 4.) Therefore, this component was also added to the base of elements. 

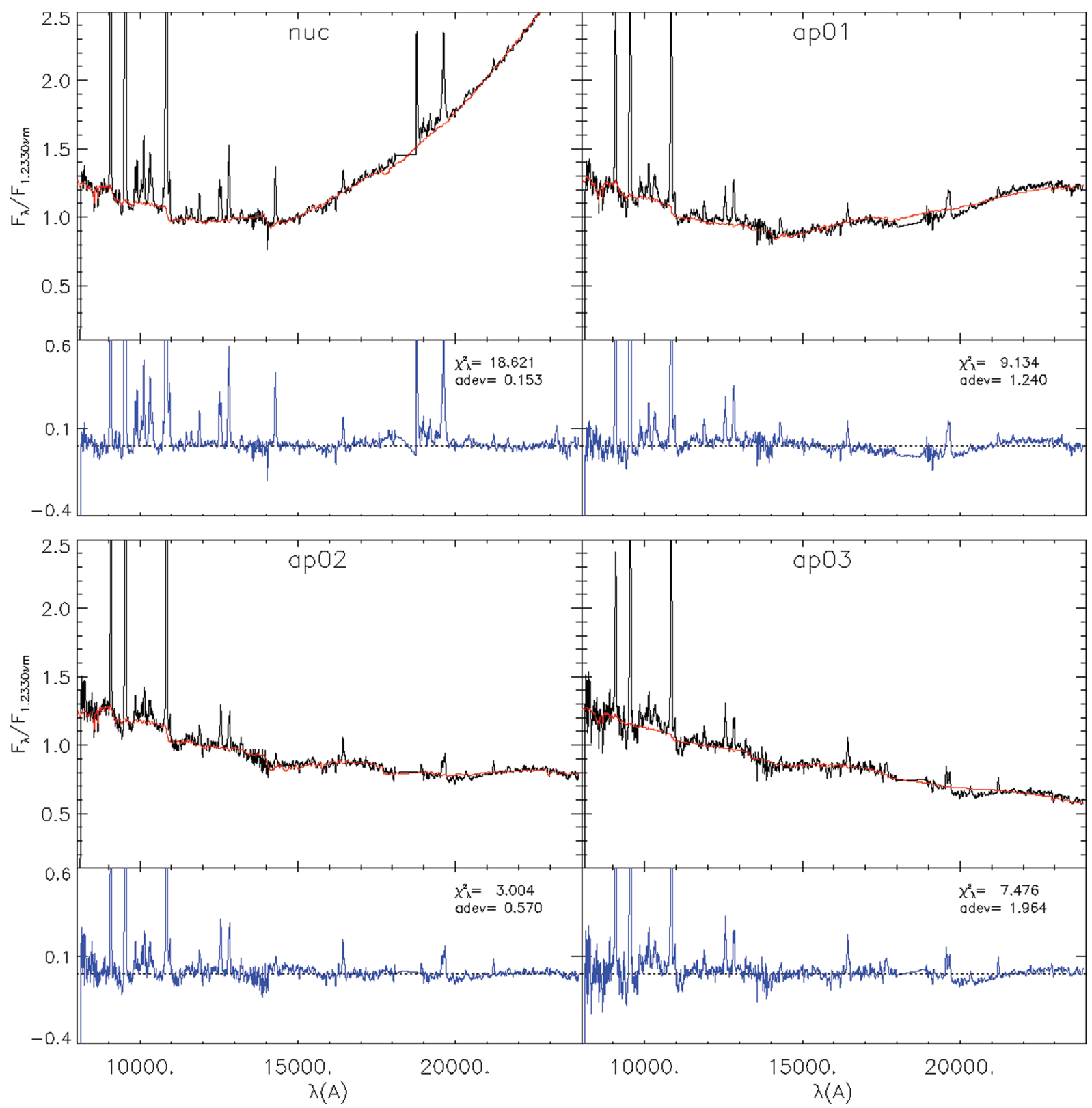

Figure 2. Synthesis results for each aperture of NGC 1068.

Besides that, the thermal emission from hot dust plays an important role in the continuum emission studied in this paper (see fig. 3 of Riffel et al. 2009). Previous studies report a minimum in the continuum emission around $1.2 \mu \mathrm{m}$ probably associated with the end of the optical continuum related to the central engine and the onset of the emission due to reprocessed nuclear radiation by dust (Barvainis 1987; Thompson 1995; Rudy et al. 2000; RodríguezArdila \& Viegas 2003; Riffel, Rodríguez-Ardila \& Pastoriza 2006; Rodríguez-Ardila \& Mazzalay 2006). This is obvious in the nuclear region but also visible in the off-nuclear apertures (see Figs 2-5). Besides that, studies like Galliano et al. (2003) have already mentioned the importance of the contribution of dust cloud emission to the continuum. In order to properly account for this component, we have included in our spectral base eight Planck distributions (blackbody, BB), with $T$ ranging from 700 to $1400 \mathrm{~K}$, in steps of $100 \mathrm{~K}$ (for more details see Riffel et al. 2009).
Extinction is modelled by STARLIGHT as due to foreground dust, and parametrized by the $V$-band extinction $A_{V}$. First, we use the Cardelli, Clayton \& Mathis (1989, hereafter CCM) extinction law, but we also compared results with the Calzetti et al. (2000, HZ5) law.

\section{SYNTHESIS RESULTS AND DISCUSSIONS}

The spectral synthesis fitting procedure is shown in Figs 2-5 for all apertures, using the CCM extinction law. For each aperture, the top panel shows the observed and synthetic spectra normalized to unit at $1.223 \mu \mathrm{m}$ (in black). The red line represents the model. The bottom panel shows the residual spectrum. The quality of the fits are measured by $\chi_{\lambda}^{2}$ and adev as defined in Cid Fernandes et al. (2004) and Riffel et al. (2009). $\chi_{\lambda}^{2}$ is the $\chi^{2}$ divided by the number of $\lambda$ values used in the fit and adev gives the percentage mean deviation 

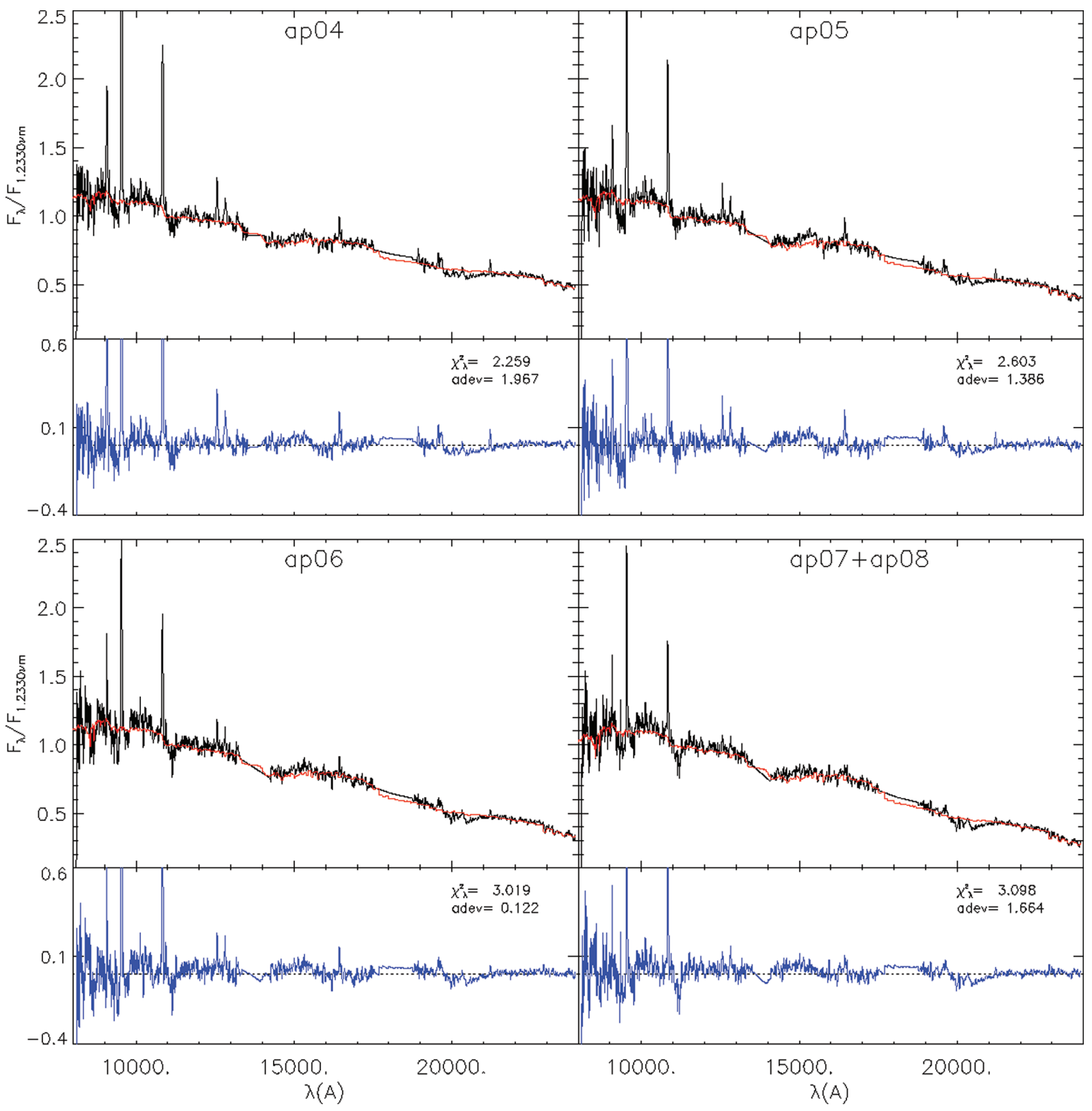

Figure 3. Synthesis results for each aperture of NGC 1068.

$\left|\mathrm{O}_{\lambda}-\mathrm{M}_{\lambda}\right| / \mathrm{O}_{\lambda}$ over all fitted pixels, where $\mathrm{O}_{\lambda}$ is the observed spectra and $\mathrm{M}_{\lambda}$ is the model spectra obtained by the synthesis.

Apertures 07 and 08 to the south and 15 and 16 to the north were too noisy, and for this reason they were added to form two larger apertures in order to improve the quality of the fit.

Following Cid Fernandes et al. (2005b), we present our results using a condensed population vector to take into account noise effects that dump small differences between similar spectral components. This is obtained by binning the population vector $\boldsymbol{x}$ into young $\left(x_{\mathrm{Y}}: t \leq 5 \times 10^{7} \mathrm{yr}\right)$, intermediate-age $\left(\boldsymbol{x}_{\mathrm{I}}: 1 \times 10^{8} \leq\right.$ $\left.t \leq 2 \times 10^{9} \mathrm{yr}\right)$ and old $\left(x_{\mathrm{O}}: t>2 \times 10^{9} \mathrm{yr}\right)$ components, using the flux distributions. The condensed vectors are plotted on the top panel of Fig. 6 for each aperture, as a function of distance to the centre. Negative distances represent the north direction and positive distances represent the south. Following Riffel et al. (2009) we have also binned the blackbody components into two compo- nents: cool $\left(\mathrm{BB}_{\mathrm{c}}, T \leq 1000 \mathrm{~K}\right)$ and hot $\left(\mathrm{BB}_{\mathrm{h}}, T \geq 1100 \mathrm{~K}\right)$. These components are defined based on the sublimation temperatures of silicate $(\sim 1000 \mathrm{~K})$ and graphite $(\sim 1200 \mathrm{~K})$ grains (Barvainis 1987; Granato \& Danese 1994). Condensed blackbody components for each aperture are also plotted in Fig. 6 as a thick red line $\left(\mathrm{BB}_{\mathrm{h}}\right)$ and dotted magenta $\left(\mathrm{BB}_{\mathrm{c}}\right)$. The contribution of the power law is also shown (black line).

The spectral synthesis shows that the overall contribution of the stellar population to the spectra is important and in many regions it contributes with more than 50 per cent of the light. The intermediateage population seems to be specially important. Indeed, one has to keep in mind that this wavelength range is specially sensible to this population. Thatte et al. (1997), for instance, found a $\sim 50 \mathrm{pc}$ stellar core centred on the $K$-band emission peak. With our data we are unable to reach that spatial scale but we could find indirect evidence confirming this result as there is a clear nuclear contribution of 

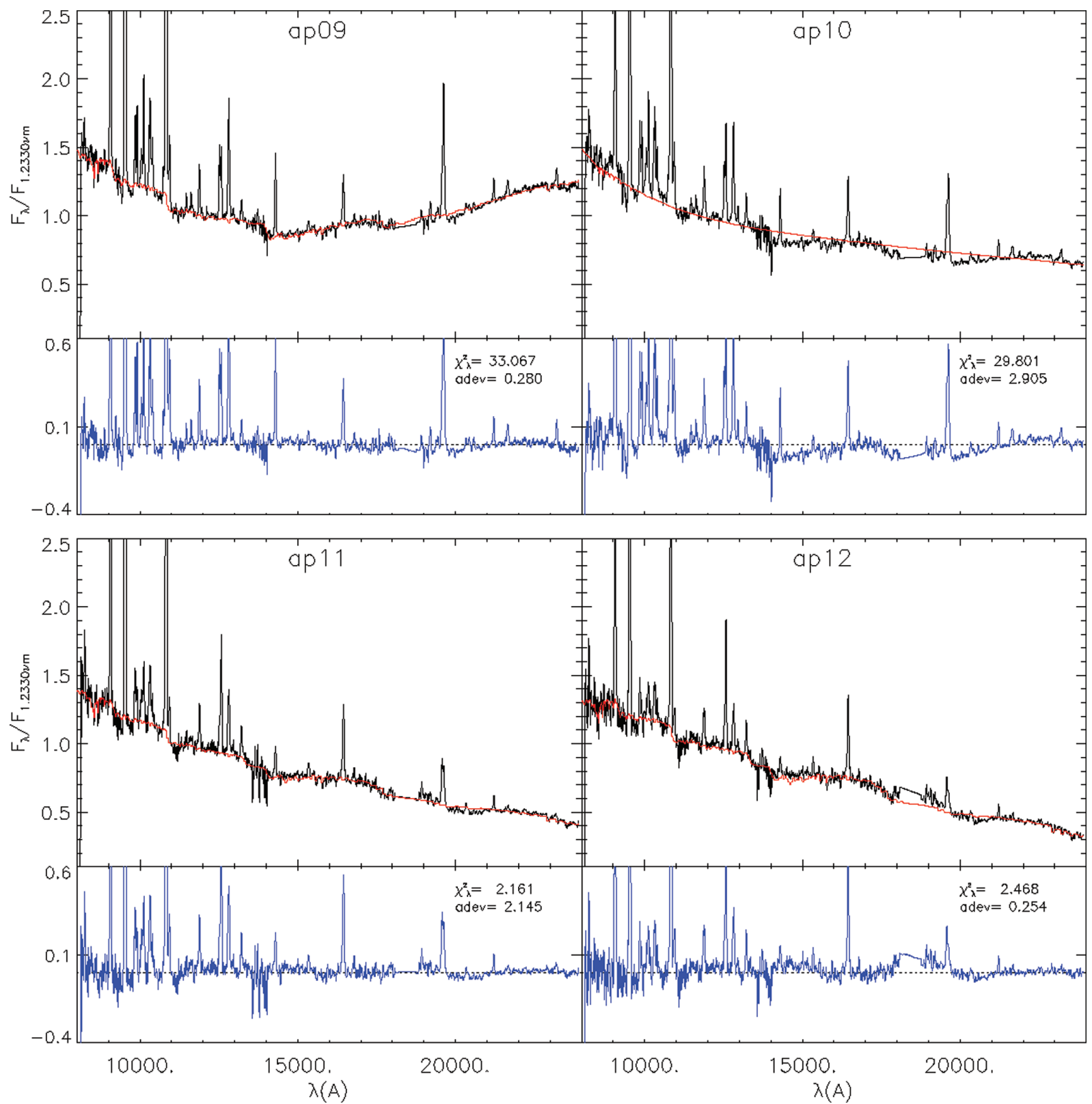

Figure 4. Synthesis results for each aperture of NGC 1068.

intermediate stellar population in a radius around $100 \mathrm{pc}$ from the nucleus. Since we are integrating along the line of sight, this population might be spread over a much larger region than $100 \mathrm{pc}$. Note that the fact that NGC 1068 is face-on minimizes this effect. Similar results are found by Davies et al. (2007). They analysed NGC 1068 among other Seyfert galaxies, using NIR adaptive optics integral field spectrograph SINFONI, and found that stellar light profiles typically have size scales of a few tens of parsecs, where there appear to have been recent, but no longer active starbursts. Results of Riffel et al. (2009) agree with this scenario: Seyferts do contain a substantial fraction of intermediate-age stellar populations.

In the optical region, Cid Fernandes et al. (2004) applied stellar population synthesis to NGC 1068 . They found the following values for the contribution of each component to the nuclear spectrum: $\mathrm{FC}=23$ per cent, $\boldsymbol{x}_{\mathrm{Y}}=24$ per cent, $\boldsymbol{x}_{\mathrm{I}}=0$ per cent and $\boldsymbol{x}_{\mathrm{O}}=54$ per cent. It can be seen that FC agrees with our results although they found a much higher contribution of the young stellar population than ours and no intermediate-age population. The reasons for this apparent contradiction might be indeed due to the fact that the optical region is more sensible to young stellar populations while the NIR is better to probe intermediate-age populations. We find average ages in this region from 1 to $19 \times 10^{8} \mathrm{yr}$, consistent with the ages Cid Fernandes et al. (2004) obtained for the stellar core (between 5 and $16 \times 10^{8} \mathrm{yr}$ ).

Perhaps our most interesting result is the small young stellar population contribution found by our stellar synthesis around $\sim 100$ pc south of the nucleus. This result, however, needs to be taken with caution because it is a common problem in the study of the stellar populations of active galaxies that a reddened young starburst (age $\leq 5 \mathrm{Myr}$ ) is indistinguishable from an AGN-type continuum (e.g. Cid Fernandes \& Terlevich 1995; Storchi-Bergmann et al. 2000). In order to test this possibility, we plot in the middle 


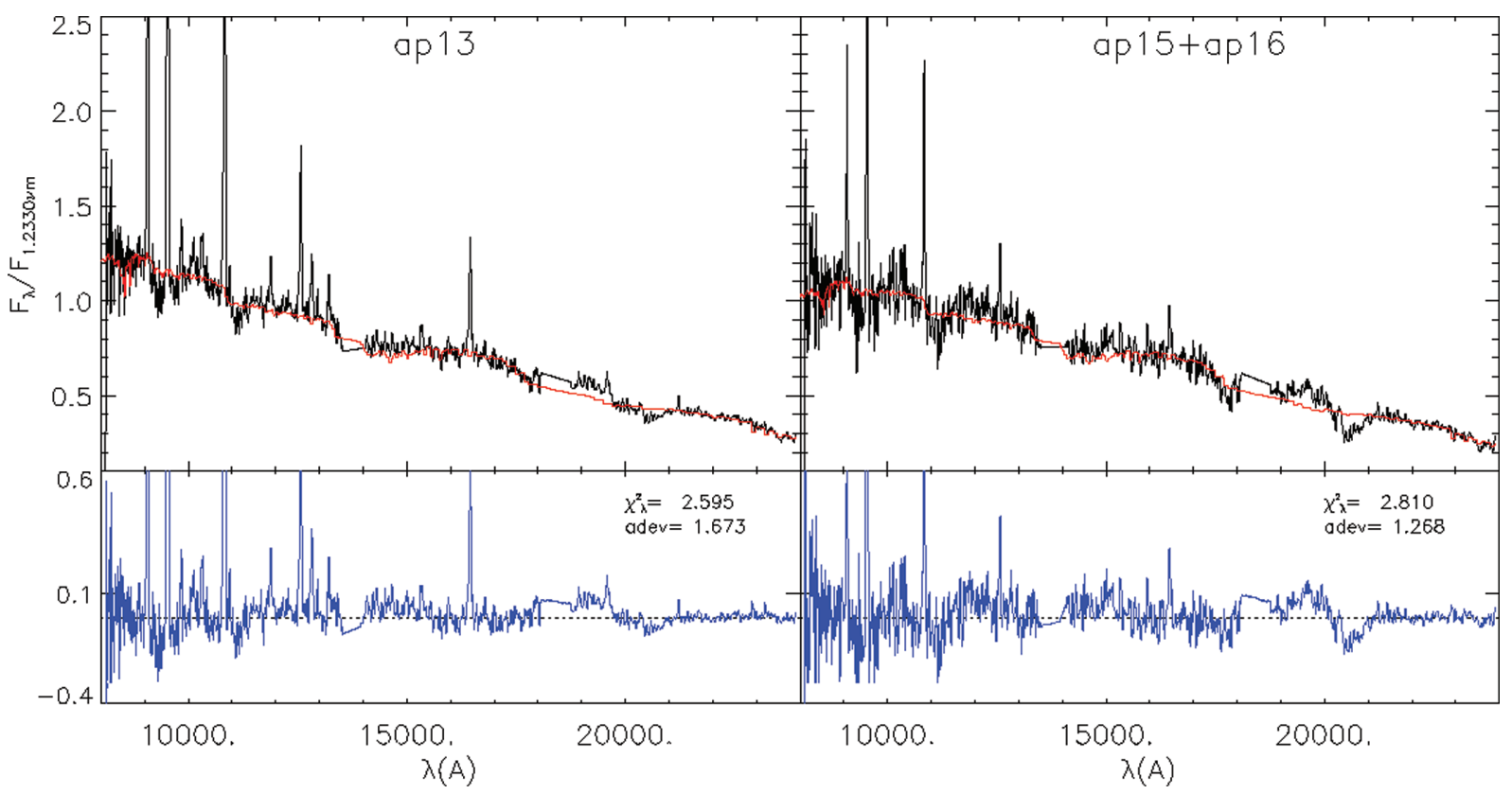

Figure 5. Synthesis results for each aperture of NGC 1068.

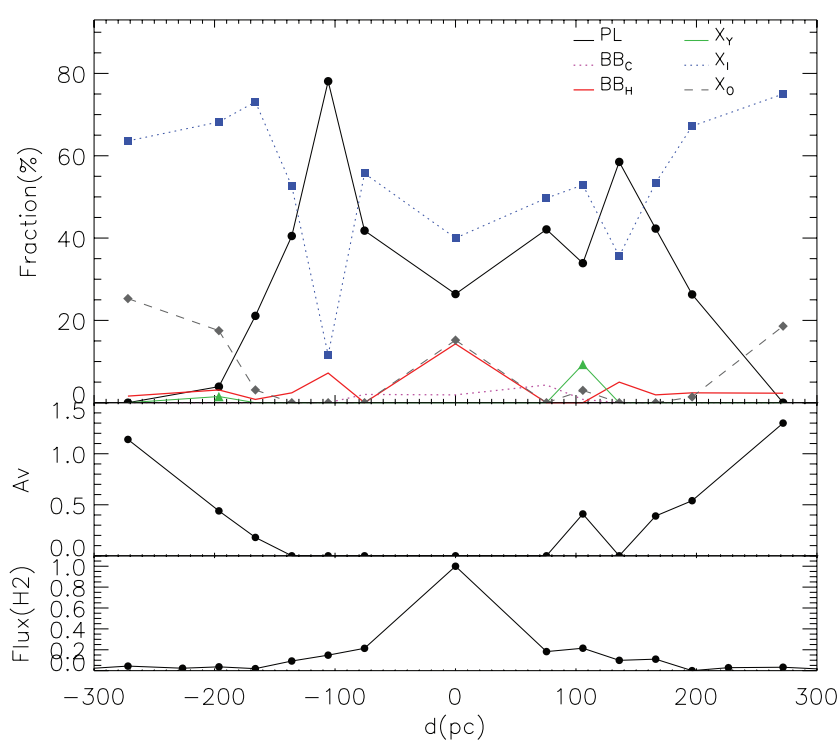

Figure 6. Synthesis results as a function of distance to the nucleus. Negative distances represent the north direction and positive distances the south. The top panel shows the flux fraction of stellar population: $x_{Y}$ (young component), $x_{\mathrm{I}}$ (intermediate-age component) and $\boldsymbol{x}_{\mathrm{O}}$ (old component), the flux fraction of hot dust (BB_H), cold dust (BB_C) and the power-law component (PL). The middle panel shows the $A_{V}$ extinction obtained by the synthesis for each aperture. The bottom panel shows the normalized $\mathrm{H}_{2} \lambda 2.12 \mu \mathrm{m}$ flux obtained in Paper I. For these results the extinction law used was from CCM.

panel of Fig. 6 the $A_{V}$ extinction found by the synthesis for each aperture. It can be seen that the young population is associated with higher extinction. It means that based only on the synthesis results we cannot state if the young stellar component is indeed present. However, other results mentioned along this section (hot dust and $\mathrm{H}_{2}$ distribution) reinforce that it is indeed a real detection.

One way to characterize the stellar population content of a galaxy by a single parameter is the mean age, defined in two ways: the first

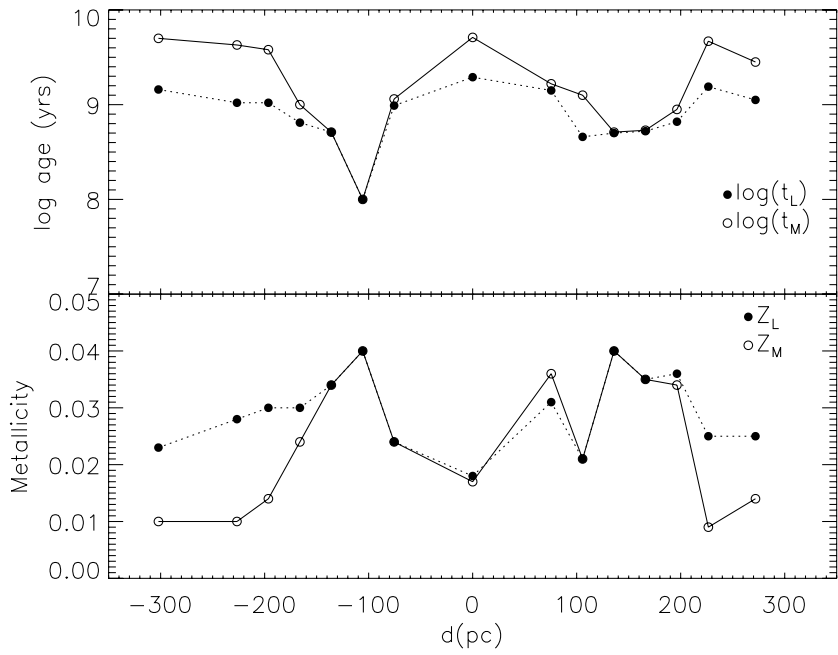

Figure 7. Results for mean age and metallicity as a function of distance to the centre. Top panel shows the light-weighted (filled) and mass-weighted (open) mean ages. Bottom panel shows the light-weighted (filled) and massweighted (open) mean metallicities. Negative distances represent the north direction and positive distances represent the south.

is weighted by light fraction and the second is weighted by the stellar mass. Both definitions are limited by the age range used in our element base (FC and BB components are excluded from the sum). The mean stellar ages derived with both definitions for each aperture are presented on the top panel of Fig. 7.

The light-weighted mean age of the apertures is biased to an intermediate-/old-age stellar population, while for the massweighted the old population clearly dominates. As stated by Cid Fernandes et al. (2005b) and Riffel et al. (2009), the mass-weighted mean age is a more physical parameter, but it has a much less direct relation with the observables. Although the mass fraction of a young stellar population might be small, it is much more luminous. Thus their contribution to the luminosity is much higher. 
A secondary parameter to describe the stellar population is the metallicity, also defined as light- and mass-weighted mean metallicity. Both definitions are bounded by the range of $Z$ used in the base. Results for the metallicity are also presented in the bottom panel of Fig. 7. These results point out to a mean value around solar for both definitions, but the light-weighted average gives higher values for the mean metallicity than the mass-weighted average. Again, the light-weighted values are more sensitive to the younger components, while the mass-weighted results are more sensitive to the older components. This result is consistent with a galaxy chemical enrichment scenario, in which the young population is enriched by the evolution of the early massive stars.

The power-law component is important in the nucleus, as expected, contributing with about 25 per cent of the light. Cid Fernandes \& Terlevich (1995) predicted that a broad component in $\mathrm{H} \beta$ becomes distinguishable whenever the scattered FC contributes with $\geq 20$ per cent to the optical continuum light. Broad permitted lines are indeed observed in the spectrum measured in polarized light, confirming that this galaxy harbours a Seyfert 1 nucleus (Antonucci \& Miller 1985; Miller et al. 1991; Antonucci, Hurt \& Miller 1994; Inglis et al. 1995; Alexander, Ruiz \& Hough 1999). Interestingly though, this is not the region where the power law dominates the spectra. There are peaks around $100-150 \mathrm{pc}$ from each side of the centre, where the power law is responsible for more than 50 per cent of the light. These peaks are probably related to shocked region. In Fig. 1, these regions correspond to apertures 03 and 10 . Aperture 03 , for example, is coincident with a region where the jet seems to be changing directions, probably due to the interaction with the ISM. If these are the regions where the jet encounters dense clouds, shocks might have an important contribution to the continuum. Another possibility is that these peaks are associated with reflected light from the broad line region, due to the higher concentration of dust. In Paper I, Martins et al. show that many emission lines have double-peaked profiles, and the maxima of the second components are also in this region. As mentioned above, it is important to keep in mind that the results for the power-law component might have uncertainties due to the fact that it cannot be distinguished from a young stellar component with dust extinction.

Hot dust also has a significant contribution to the continuum. It has a peak in the nuclear region, which, as reported by Thatte et al. (1997), is a strong evidence of a dense circumnuclear torus. It also has peaks in the regions where the power law has a maximum, around $100 \mathrm{pc}$ from the centre on both sides. This is again another indication that these are regions where the jet finds dense dusty/molecular clouds. As a further indication of this hypothesis, we plot in bottom panel of Fig. 6 the intensity of the $\mathrm{H}_{2} \lambda 2.12 \mu \mathrm{m}$ emission line, measured in Paper I. This line probes the conditions of the 'warm' molecular gas, and can be originated from photodissociated regions, emission due to shocks or X-ray-heated gas. It is interesting to note that this emission line shows extended emission around the same region where the hot dust and the young stellar population are found.

Although only a small flux fraction was detected, cold dust also has an interesting distribution. It appears to be concentrated on the southern spectra. It was already observed that [O III] emission is very faint in the south-west radio cone direction (Unger et al. 1992) and very little UV/optical polarized light (Scarrott et al. 1991) in this region. This suggests that the south-west cone lies behind the plane of the galaxy and is extinguished by the dust in the plane (e.g. Gallimore et al. 1994; Bland-Hawthorn et al. 1997), in agreement with the cool dust distribution we find here.

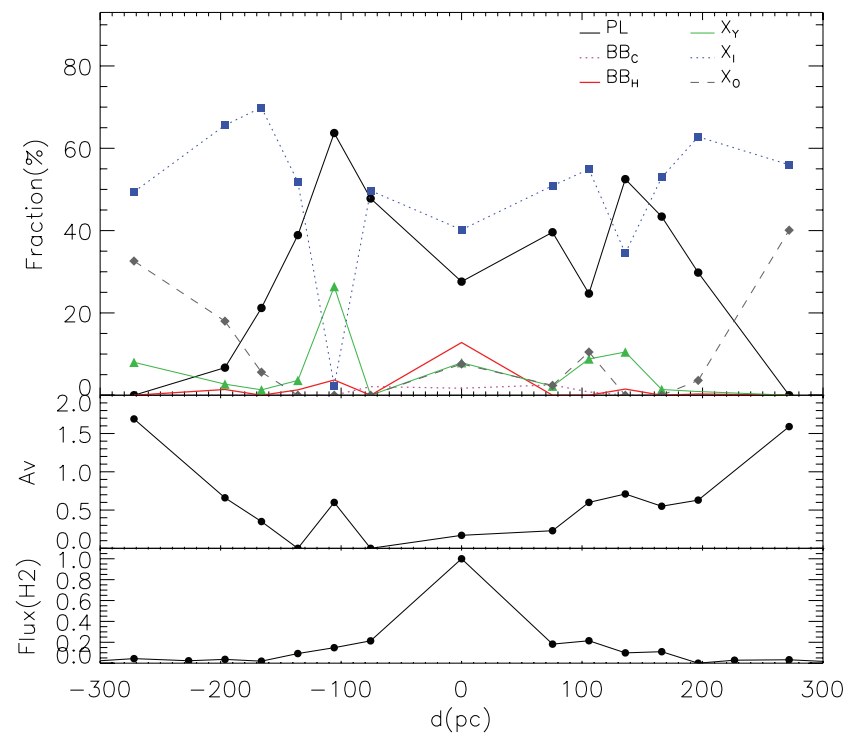

Figure 8. Same as in Fig. 6, but for Calzetti's extinction law.

We also calculated the hot dust mass, obtaining a total value of $3.4 \times 10^{-2} \mathrm{M}_{\odot}$. From this value, 96 per cent is from the central aperture, and about 3 per cent are from apertures 01,02 and 09. These are apertures where the contribution of $\mathrm{BB}_{\mathrm{C}}$ is higher. For the other apertures, $\mathrm{BB}_{\mathrm{H}}$ is the only one present. One has to keep in mind that, although the luminosity fraction of cold dust is small, their emissivity is also small, so it requires a large mass. On the other hand, the emissivity for very hot grains is large, so the calculated mass is small. It is interesting to note, however, that the total mass calculated for the nuclear aperture is one of the highest mass values for Seyfert galaxies determined so far (see table 5 of Riffel et al. 2009).

We tested if our results were dependent of some of the choices we made for the synthesis. For example, in Fig. 8 we show results for the synthesis using Calzetti's extinction law instead of CCM. Using this extinction law the contribution of the stellar population is even clearer, and it is present in both sides of the nucleus.

We also tested modifying the power-law index to 1.25 and 1.75 to check if the FC contribution would change. We find that our results are not affected by this change.

\section{SUMMARY AND CONCLUSIONS}

We investigated the NIR spectra of NGC 1068 extended across 15 $\operatorname{arcsec}(\sim 1100 \mathrm{pc})$ in the north-south direction. The spectra were taken with the IRTF SpeX instrument, obtained in the SXD. The objective was to disentangle the galaxy's spectral energy distribution components along the full wavelength coverage $(0.8-2.4 \mu \mathrm{m})$ using stellar population synthesis. This was done here for the first time for this galaxy. We used the STARLIGHT code, which considers the whole observed spectrum, continuum and absorption features.

We find that intermediate-age stellar population contributes significantly to the continuum, especially in the inner $200 \mathrm{pc}$. We do not have the spatial resolution to determine the size of this nuclear stellar cluster, but results agree with previous determinations of the stellar population of NGC 1068 from Davies et al. (2007) and Riffel et al. (2009).

We find a small contribution of a young stellar population component about $100 \mathrm{pc}$ from the nucleus. Although this result has to be considered carefully, since the detection might be an artefact 
from the method used, taken together with our other findings this suggests that this is the region where the jet interacts with the ISM and might be forming stars.

The contribution of a power-law continuum in the centre is about 25 per cent, which is expected from a scattered Seyfert 1 nucleus (Cid Fernandes \& Terlevich 1995). We also find peaks in the contribution of the FC about 100-150 pc from the nucleus, both in the north and in the south direction. These might be associated with the region where the jet encounters dense clouds. This result is further supported by peaks of hot dust found around the same regions. We compared these results with the $\mathrm{H}_{2}$ emission-line profile obtained from Paper I. This line also shows extended emission around the same region where the hot dust is found. Taking this together with the young stellar population detection, we believe there is strong evidence that the interaction of the jet with the dense ISM in NGC 1068 is forming stars. Further investigation with more spatial resolution is needed to confirm this hypothesis.

Hot dust also has an important contribution to the nuclear region, which reinforces the idea of the presence of a dense, circumnuclear torus in this galaxy. Cold dust appears mostly in the south direction, which supports the idea that the south-west emission is behind the plane of the galaxy, and is extinguished by the dust in the plane. We determine the dust mass in this galaxy to be about $3.4 \times 10^{-2} \mathrm{M}_{\odot}$, which puts NGC 1068 as one of the Seyferts with the highest dust content measured.

\section{ACKNOWLEDGMENTS}

The authors thank the anonymous referee for valuable comments. This research has been partially supported by the Brazilian agency FAPESP (2007/04316-1). RR thanks the Brazilian funding agency CAPES. AR-A thanks to CNPq for financial support through grant 308877/2009-8. LPM and AR-A were Visiting Astronomers at the Infrared Telescope Facility, which is operated by the University of Hawaii under Cooperative Agreement no. NNX-08AE38A with the National Aeronautics and Space Administration, Science Mission Directorate, Planetary Astronomy Program.

\section{REFERENCES}

Alexander D. M., Ruiz M., Hough J. H., 1999, in Laureijs R. J., Siebenmorgen R., eds, ESA-SP 435, Workshop on ISO Polarization Observations. ESA, Noordwijk, p. 1

Alloin D., Pantin E., Lagage P. O., Granato G. L., 2000, A\&A, 363, 926

Antonucci R. R. J., Miller J. S., 1985, ApJ, 297, 621

Antonucci R. R. J., Hurt T., Miller J. S., 1994, ApJ, 430, 210

Asari N. V., Cid Fernandes R., Stasińska G., Torres-Papaqui J. P., Matheus A., Sodré L., Schoenell W., Gomes J. M., 2007, MNRAS, 381, 263

Barvainis R., 1987, ApJ, 320, 537

Calzetti D., Armus L., Bohlin R. C., Kinney A. L., Koornneef J., StorchiBergmann T., 2000, ApJ, 533, 682

Cardelli J. A., Clayton G. C., Mathis J. S., 1989, ApJ, 345, 245 (CCM)

Cid Fernandes R., Terlevich R., 1995, MNRAS, 272, 423

Cid Fernandes R., Gu Q., Melnick J., Terlevich E., Terlevich R., Kunth D., Rodrigues Lacerda R., Joguet B., 2004, MNRAS, 355, 273
Cid Fernandes R., Matheus A., Sodré L., Stasiśka G., Gomes J. M., 2005a, MNRAS, 358, 363

Cid Fernandes R., González Delgado R. M., Storchi-Bergmann T., Martins L. P., Schmitt H., 2005b, MNRAS, 356, 270

Cid Fernandes R. et al., 2009, Rev. Mex. Astron. Astrofís., 35, 127

Davies R. I., Sánchez F. M., Genzel R., Tacconi L. J., Hicks E. K. S., Friedrich S., Sternberg A., 2007, ApJ, 671, 1388

Fathi K., Storchi-Bergmann T., Riffel R. A., Schimdt A. A., 2006, ApJ, 641, 25

Galliano E., Alloin D., Granato G. L., Villar-Martín M., 2003, A\&A, 412, 615

Gallimore J. F., Baum S. A., O'Dea C. P., Brinks E., Pedlar A., 1994, ApJ, 422,13

Gallimore J. F., Baum S. A., O’Dea C. P., Pedlar A., 1996, ApJ, 458, 136

Granato G. L., Danese L., 1994, MNRAS, 268, 235

Inglis M. D., Young S., Hough J. H., Gledhill T., Axon D. J., Bailey J. A., Ward M. J., 1995, MNRAS, 275, 398

Knapen J. H., Shlosman I., Peletier R. F., 2000, ApJ, 529, 93

Koski A. T., 1978, ApJ, 223, 56

Lançon A., Wood P. R., 2000, A\&AS, 146, 217

Macchetto F., Capetti A., Sparks W. B., Axon D. J., Boksenberg A., 1994, ApJ, 435, 15

Maiolino R., Ruiz M., Rieke G. H., Papadopoulos P., 1997, ApJ, 485, 552

Maraston C., 2005, MNRAS, 362, 799

Martins L. P., Rodríguez-Ardila A., de Souza R., Gruenwald G., 2010, MNRAS, in press (doi:10.1111/j.1365-2966.2010.17042.x) (Paper I, this issue)

Mateus A., Sodré L., Cid Fernandes R., Stasińska G., Schoenell W., Gomes J. M., 2006, MNRAS, 370, 721

Miller J. S., Goodrich R. W., Mathews W. G., 1991, ApJ, 378, 47

Origlia L., Oliva E., 2000, New Astron. Rev., 44, 257

Rayner J. T., Toomey D. W., Onaka P. M., Denault A. J., Stahlberger W. E., Vacca W. D., Cushing M. C., Wang S., 2003, PASP, 155, 362

Rees M. J., 1989, MNRAS, 239, 1

Riffel R., Rodríguez-Ardila A., Pastoriza M. G., 2006, A\&A, 457, 61

Riffel R., Pastoriza M. G., Rodríguez-Ardila A., Maraston C., 2007, ApJ, 659,103

Riffel R. A., Storchi-Bergmann T., Winge C., McGregor P. J., Beck T., Schmitt H., 2008, MNRAS, 385, 1129

Riffel R., Pastoriza M. G., Rodríguez-Ardila A., Bonatto C., 2009, MNRAS, 400, 273

Rodríguez-Ardila A., Mazzalay Z., 2006, MNRAS, 367, L57

Rodríguez-Ardila A., Viegas S. M., 2003, MNRAS, 340, 33

Rudy R. J., Mazuk S., Puetter R. C., Hamann F., 2000, ApJ, 539, 166

Scarrott S. M., Rolph C. D., Wolstencroft R. W., Tadhunter C. N., 1991, MNRAS, 249, 16

Shlosman I., Frank J., Belgmann M. C., 1989, Nat, 338, 45

Shlosman I., Belgmann M. C., Frank J., 1990, Nat, 345, 679

Simpson J. P., Colgan S. W. J., Erickson E. F., Hines D. C., Schultz A. S. B., Trammell S. R., 2002, ApJ, 574, 95

Storchi-Bergmann T., Raimann D., Bica E. L. D., Fraquelli H. A., 2000, ApJ, 544, 747

Thatte N., Quirrenbach R., Maiolino R., Tecza M., 1997, ApJ, 490, 238

Thompson R. J. Jr, 1995, ApJ, 454, 660

Telesco C. M., Decher R., 1988, ApJ, 334, 573

Unger S. W., Lewis J. R., Pedlar A., Axon D. J., 1992, MNRAS, 258, 371

Wilson A. S., Ulvestad J. S., 1983, ApJ, 275, 8

This paper has been typeset from a $\mathrm{T}_{\mathrm{E}} \mathrm{X} / \mathrm{L} \mathrm{T}_{\mathrm{E}} \mathrm{X}$ file prepared by the author. 